\title{
Surface structures and growth kinetics of InGaN(0001) grown by molecular beam epitaxy
}

Huajie Chen, R. M. Feenstra, J. E. Northrup, T. Zywietz, J. Neugebauer, and D. W. Greve

Citation: Journal of Vacuum Science \& Technology B: Microelectronics and Nanometer Structures Processing, Measurement, and Phenomena 18, 2284 (2000); doi: 10.1116/1.1306296

View online: https://doi.org/10.1116/1.1306296

View Table of Contents: https://avs.scitation.org/toc/jvn/18/4

Published by the American Institute of Physics

\section{ARTICLES YOU MAY BE INTERESTED IN}

Control of GaN surface morphologies using plasma-assisted molecular beam epitaxy Journal of Applied Physics 88, 1855 (2000); https://doi.org/10.1063/1.1305830

Phase separation in InGaN thick films and formation of InGaN/GaN double heterostructures in the entire alloy composition

Applied Physics Letters 70, 1089 (1997); https://doi.org/10.1063/1.118493

A growth diagram for plasma-assisted molecular beam epitaxy of In-face InN Journal of Applied Physics 102, 064907 (2007); https://doi.org/10.1063/1.2781319

In/GaN(0001)- $(\sqrt{3} \times \sqrt{3}) R 30^{\circ}$ adsorbate structure as a template for embedded (In, Ga)N/GaN monolayers and short-period superlattices

Applied Physics Letters 110, 072104 (2017); https://doi.org/10.1063/1.4976198

Solid phase immiscibility in GalnN

Applied Physics Letters 69, 2701 (1996); https://doi.org/10.1063/1.117683

Homoepitaxial growth of GaN under Ga-stable and N-stable conditions by plasma-assisted molecular beam epitaxy

Journal of Applied Physics 82, 5472 (1997); https://doi.org/10.1063/1.365575 


\title{
Surface structures and growth kinetics of $\ln G a N(0001)$ grown by molecular beam epitaxy
}

\author{
Huajie Chen and R. M. Feenstra ${ }^{a}$ \\ Department of Physics, Carnegie Mellon University, Pittsburgh, Pennsylvania 15213 \\ J. E. Northrup \\ Xerox Palo Alto Research Center, 3333 Coyote Hill Road, Palo Alto, California 94304 \\ T. Zywietz and J. Neugebauer \\ Fritz-Haber-Institut der Max-Planck-Gesellschaft, Faradayweg 4-6, D-14195 Berlin, Germany \\ D. W. Greve \\ Department of Electrical and Computer Engineering, Carnegie Mellon University, Pittsburgh, \\ Pennsylvania 15213
}

(Received 6 April 2000; accepted 26 May 2000)

\begin{abstract}
The surface structures and growth kinetics of $\operatorname{InGaN}(0001)$ are studied. It is well known that during molecular beam epitaxy GaN surfaces undergo a smooth to rough transition when the growth condition is switched from $\mathrm{Ga}$ rich to $\mathrm{N}$ rich. It is found here that indium atoms have only a small effect on this transition when deposited on $\mathrm{GaN}(000 \overline{1})$, but when deposited on $\mathrm{GaN}(0001)$ the indium acts as a surfactant and greatly extends the regime of smooth growth. Near the smooth/rough transition of $\operatorname{InGaN}(0001)$ growth, a bright $\sqrt{3} \times \sqrt{3}$ reconstruction is observed at growth temperature. The formation kinetics of this reconstruction are studied in detail. Scanning tunneling microscopy and total energy computations are used to study the structure of $\operatorname{InGaN}(0001)$ surfaces under metal rich conditions. Indium is found to occupy the top two atomic layers of the crystal; its incorporation in the second layer produces significant strain, leading to the formation of small pits on the surface and increased indium concentration inside and around the pits. (C) 2000 American Vacuum Society. [S0734-211X(00)07004-9]
\end{abstract}

\section{INTRODUCTION}

$\mathrm{In}_{x} \mathrm{Ga}_{1-x} \mathrm{~N}$ alloys have recently attracted great interest because of the successful development of InGaN/GaN based blue light-emitting devices and lasers, where $\mathrm{InGaN}$ is used as the active layer. ${ }^{1}$ While the growth of high quality material and fabrication of devices have been successful, fundamental understanding of the growth, structure, and luminescence mechanism is far from complete. Issues such as indium compositional fluctuations ${ }^{2}$ and an apparent surfactant effect due to indium ${ }^{3}$ were investigated recently. These issues are explored here by an extensive study of the surface structure and growth kinetics of InGaN.

The basic surface structures discussed here are illustrated in Fig. 1, for the limit of very In-rich conditions. Figure 1(a) shows the previously determined structure for the N-polar InGaN $(000 \overline{1})$ surface, consisting of a monolayer (ML) of In bonded to a GaN bilayer. ${ }^{4}$ For less In-rich conditions this monolayer contains a mixture of In and Ga. For the Ga-polar $\operatorname{InGaN}(0001)$ surface, prior theoretical studies indicate that the surface consists of either a single ML of In in the topmost layer, or one ML in the top layer plus another ML in the second layer. ${ }^{5}$ The latter case is illustrated in Fig. 1(b). Following the notation of Ref. 5 we refer to these layers as A1 and S3, as indicated in Fig. 1(b). As discussed below, we find that the S3 layer is actually only partially occupied by

a)Electronic mail: feenstra@andrew.cmu.edu
In. Furthermore, we observe the formation of an array of small pits (vacancy islands) on the surface.

This article consists of three parts. First, we present a detailed study of the effect of indium atoms on the GaN surface morphology. It is well known that during molecular beam epitaxy (MBE) growth of GaN the surface undergoes smooth to rough transition when the growth condition is switched from $\mathrm{Ga}$ to $\mathrm{N}$ rich. ${ }^{6-8}$ Here, we find that indium atoms have only a small effect on this smooth/rough transition when deposited on $\mathrm{GaN}(000 \overline{1})$, but when indium is deposited on $\mathrm{GaN}(0001)$ the gallium flux can be reduced greatly before the growth becomes rough. Second, a $\sqrt{3}$ $\times \sqrt{3}$ reconstruction is observed during $\operatorname{InGaN}(0001)$ growth near the smooth/rough transition point. This reconstruction thus serves as a useful real-time indicator during growth of when the morphology is near the smooth/rough transition, and we have studied the formation kinetics of the reconstruction in an effort to determine its structure. Finally, In$\mathrm{GaN}(0001)$ surfaces under metal rich conditions were studied using scanning tunneling microscopy (STM) and total energy computations. We find that the indium atoms occupy the top two surface layers. Based on theoretical results for the surface structures, we argue that strain arising from the presence of In in the second (S3) layer leads to the formation of small pits on the surface. Inside, and at the border of the pits, the surface concentration of indium is found to be larger than that far from the pits, which may lead to inhomogeneous indium incorporation in the bulk film. 


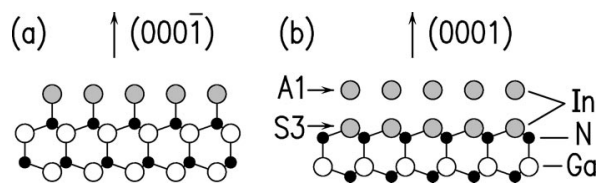

FIG. 1. Basic structure of In-rich InGaN surfaces: (a) (000) $)$ and (b) (0001). A1 and S3 layers are indicated in (b).

\section{EXPERIMENT}

The studies described here were performed in a combined $\mathrm{MBE} /$ surface analysis system. The growth chamber contains gallium and indium effusion cells, a rf-plasma nitrogen source, and a reflection high energy electron diffraction (RHEED) system. GaN(0001) was grown on Si-face 6H$\mathrm{SiC}(0001)$ substrates, with the polish damage removed by hydrogen etching. ${ }^{9}$ After the hydrogen etching, the substrate was introduced into the growth chamber and outgassed up to the temperature of $800^{\circ} \mathrm{C}$. A few monolayers of Si were deposited onto the surface and the substrate was then annealed to about $1000{ }^{\circ} \mathrm{C}$ until a $\sqrt{3} \times \sqrt{3}$ reconstruction was obtained. ${ }^{10} \mathrm{GaN}$ was directly grown on this surface at growth temperature of $670{ }^{\circ} \mathrm{C}$. $\mathrm{GaN}(000 \overline{1})$ was also grown at $670^{\circ} \mathrm{C}$, on sapphire substrates, with pregrowth nitridation of the substrate performed at $950{ }^{\circ} \mathrm{C}$ and using a lowtemperature $\mathrm{GaN}$ buffer layer grown at $500{ }^{\circ} \mathrm{C} .{ }^{4}$ Following the GaN growth with typical thickness of $200 \mathrm{~nm}$, the substrate temperature is lowered to $600-630^{\circ} \mathrm{C}$ for the $\mathrm{InGaN}$ deposition. Typical growth rates for the $\mathrm{GaN}$ and $\mathrm{InGaN}$ are $200 \mathrm{~nm} / \mathrm{h}$. Gallium and indium flux rates were calibrated with an in situ crystal thickness monitor. The nitrogen plasma source was operated at a power level of $550 \mathrm{~W}$ and pressure of $1.8 \times 10^{-5}$ Torr. The substrate temperature was measured by an optical pyrometer with emissivity set to be 0.7. After growth, the sample was quenched to room temperature, and transferred under vacuum to the analysis chamber for STM and Auger study. The base pressures of the growth and analysis chambers were in the $10^{-11}$ Torr range.

Auger spectroscopy was measured with a Perkin-Elmer $15-255 \mathrm{G}$ system. The amount of indium on the surface is determined from the indium/nitrogen peak-peak intensity ratio as measured by the Auger spectroscopy. From the known bonding arrangements for In on the surface (Fig. 1), using electron mean free paths and atomic sensitivity factors obtained from Ref. 11, and using the bulk indium composition obtained from x-ray diffraction (XRD) measurement, the amount of indium on the surface can be evaluated from a measured ratio of In $404 \mathrm{eV}$ to $\mathrm{N} 379 \mathrm{eV}$ Auger lines. However, when we apply this procedure to a surface for which the indium composition was confidently known from STM images, ${ }^{4}$ the computed result from Auger data gives only about half of the expected value. The origin of this discrepancy is not well understood at present. Nevertheless, we feel that a relative measure of the indium surface concentration between different samples should still be reasonably accurate, and that type of procedure is used here in evaluating surface coverages. In any case, our final determinations of

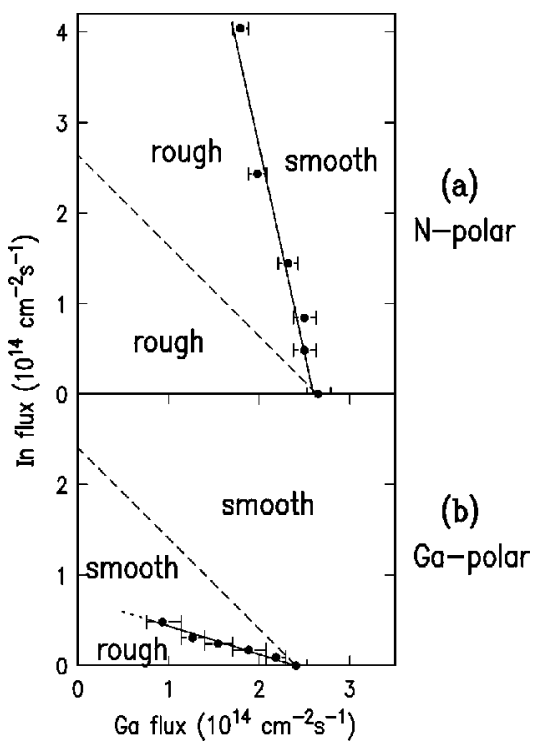

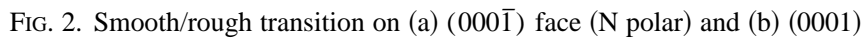
face (Ga polar). Nitrogen flux was fixed in both experiments. Substrate temperature was $600^{\circ} \mathrm{C}$. Experimental data are shown with dots, each with an error bar. A dashed line is shown in each figure for comparison denoting the line with constant total metal flux. To the right of the transition lines (solid lines) the growth is smooth.

indium coverage are made on the basis of both STM images and Auger measurements, with the Auger results mainly being used to add confidence in the interpretation of the STM images.

\section{RESULTS}

\section{A. Effect of indium on the smooth/rough transition}

During MBE growth of GaN, the surface undergoes a transition from smooth to rough morphology when the growth condition is switched from Ga to N-rich. ${ }^{6-8}$ It was previously observed that the material has high quality when grown in the smooth regime; ${ }^{6}$ it is therefore important to determine the smooth/rough transition point for $\mathrm{GaN}$ and InGaN. It has been reported that indium atoms serve as a surfactant, keeping the growth in the smooth regime when the gallium flux is slightly reduced beneath the transition flux. $^{3}$ However, in our prior growth of InGaN on $\mathrm{GaN}(000 \overline{1})$, such a surfactant effect was not observed. Instead we found that indium atoms had little effect on the smooth/rough transition, ${ }^{12}$ possibly due to the different polarity of GaN used in that case. In this work, a detailed study is carried out on the smooth/rough transition of $\mathrm{InGaN}$ growth on both the $(000 \overline{1})$ and $(0001)$ faces.

We find a dramatic difference in the smooth/rough behavior between the $(000 \overline{1})$ and (0001) faces, as shown in Figs. 2(a) and 2(b), respectively. For both experiments, the nitrogen flux was kept constant. Then, a certain indium flux was applied, and gallium flux was adjusted to find the smooth/ rough transition point. For comparison, dashed lines in Fig. 2 show where the total metal flux (indium+gallium) is constant. Figure 2(a) shows that for the $(000 \overline{1})$ face, even when a large indium flux is applied, the gallium flux can only be reduced slightly before the growth becomes rough. In contrast, for the (0001) face, it is found that when the indium 
flux is applied the gallium flux can be greatly reduced (by an amount considerably greater than that of the added indium flux) before the growth becomes rough. Thus, indium serves as a surfactant for the (0001) surface.

While this contrast between the effect of indium atoms on the surface morphology of the (0001) and (0001) faces is dramatic, it is also important to realize that in order to incorporate indium into the bulk the gallium flux must be lower than the transition flux of gallium in the absence of indium. ${ }^{4,13}$ Roughly speaking, the reason for this condition is that indium atoms tend to segregate to the surface so that when there is enough gallium the indium atoms do not incorporate. ${ }^{14}$ Therefore, in order to grow InGaN with significant indium content, growth on the $(000 \overline{1})$ face must occur in the rough growth regime whereas it can take place in the smooth regime for the (0001) face. Since one generally desires smooth morphology (implying faster surface diffusion) during growth, we conclude that the (0001) surface appears to be better suited for high quality InGaN growth.

Another implication of this surfactant effect on the (0001) surface is that more nitrogen must desorb from the surface when the gallium flux is greatly reduced in the presence of indium, since the growth is stoichiometric and now the total metal flux is lower than that during GaN growth without indium. This increased desorption could come about either as a result of higher nitrogen surface diffusivity or higher nitrogen surface concentration, where in both cases the nitrogen atoms have more chance to meet and form molecules and then leave the surface. In GaN growth, it was found that nitrogen accumulation leads to a higher diffusion barrier and hence rough growth. ${ }^{7}$ However, as will be shown in the next part of this article, with a nitrogen rich $\sqrt{3} \times \sqrt{3}$ reconstruction the growth can still be smooth, which implies that indium lowers the diffusion barrier even when the surface has high nitrogen concentration.

\section{B. $\sqrt{3} \times \sqrt{3}$ reconstruction on the $(0001)$ face}

During plasma-assisted $\mathrm{MBE}$ of $\mathrm{GaN}$, reconstructions are rarely observed at the growth temperature. Some groups have reported a $2 \times 2$ reconstruction during growth, ${ }^{15}$ although we have found previously that this structure is due to the presence of arsenic atoms in the growth environment ${ }^{16}$ (for growth using ammonia a different, intrinsic $2 \times 2$ structure occurs $\left.{ }^{17}\right)$. For the case of InGaN growth on the (0001) face, we observe a bright $\sqrt{3} \times \sqrt{3}$ reconstruction (in the absence of arsenic) at the growth temperature. This reconstruction is observed when the Ga flux is near or below the transition point between rough and smooth growth, in the presence of indium. Figure 3 shows the corresponding RHEED pattern, which is a $3 \times$ structure when viewed along (1 100$)$ azimuth and $1 \times$ structure along $(11 \overline{2} 0)$ azimuth.

We have varied the growth conditions and surface stoichiometry in an effort to determine the geometric structure of this reconstruction. For reference, various reconstructions on $\mathrm{GaN}(0001)$ at room temperature are well understood: ${ }^{18}$ there are " $1 \times 1$, ,' $6 \times 4,5 \times 5$, and $2 \times 2$ reconstructions corre-

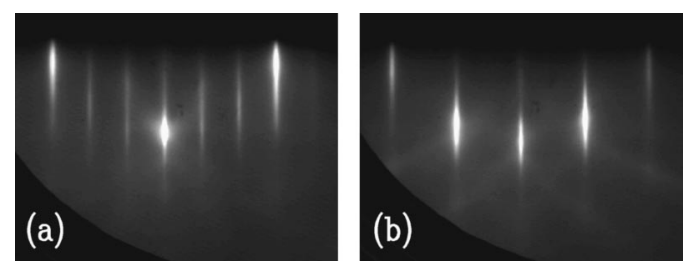

FIG. 3. RHEED pattern of $\sqrt{3} \times \sqrt{3}$ reconstruction: (a) a $3 \times$ structure when viewed along (1 100$)$ azimuth and (b) a $1 \times$ structure along (11 $\overline{2} 0)$ azimuth.

sponding to different amount of gallium atoms on the surface, with the $1 \times 1$ most gallium rich and $2 \times 2$ most gallium deficient (this $2 \times 2$ reconstruction is different than the two $2 \times 2$ structures mentioned in the previous paragraph, since it is observed only during growth interrupts). Since the $\sqrt{3}$ $\times \sqrt{3}$ reconstruction appears only when the gallium flux is reduced to near the transition point, we expect that there are not multiple Ga layers on the surface, so a first guess of the structure is an adlayer of indium with $\sqrt{3} \times \sqrt{3}$ structure on top of a gallium terminated GaN surface. Thus, in our first experimental effort, a GaN layer was grown in gallium rich growth conditions to obtain a flat surface. The surface showed a $1 \times 1$ reconstruction as usual when cooled down. Then the sample was heated to about $730^{\circ} \mathrm{C}$ for $90 \mathrm{~s}$ to desorb the excess gallium. The surface then showed a faint $2 \times 2$ reconstruction when cooled down. Then indium atoms were deposited onto this surface up to a few monolayers $\left(1 \mathrm{ML}=1.14 \times 10^{15}\right.$ atoms $/ \mathrm{cm}^{2}$ ) with a rate of $0.5-2 \mathrm{ML} /$ min. No $\sqrt{3} \times \sqrt{3}$ reconstruction was observed during the deposition. The same process was repeated with indium deposition carried out at the growth temperature, and again no $\sqrt{3} \times \sqrt{3}$ was observed, indicating that the indium adatoms on the gallium layer model is incorrect.

Based on the above results, it appears that nitrogen may play an important role in the $\sqrt{3} \times \sqrt{3}$ reconstruction. So for the next attempt, the nitrogen plasma source was turned on when indium was deposited and this time the $\sqrt{3} \times \sqrt{3}$ appeared very quickly. The same result occurred when indium was deposited during the GaN growth if the growth was in the nitrogen rich regime. For the latter case, it was found that the $\sqrt{3} \times \sqrt{3}$ was clearly seen when about 1/10 ML of indium was deposited, and the intensity of the reconstruction was maximum when about $1 / 3 \mathrm{ML}$ of indium was deposited. If the gallium flux was not too small, so that in the presence of an indium flux the gallium flux is well above the smooth/ rough transition point, then the intensity of the reconstruction will gradually decrease and finally disappear when more indium is deposited (consistent with the observation that the $\sqrt{3} \times \sqrt{3}$ can persist only near or below the transition point). Also, when GaN is grown in the gallium rich regime (without indium), then deposition of indium atoms does not lead to $\sqrt{3} \times \sqrt{3}$ reconstruction. Thus, $\mathrm{N}$-rich conditions are required for the formation of the $\sqrt{3} \times \sqrt{3}$ structure. In the extreme case, the $\sqrt{3} \times \sqrt{3}$ reconstruction was found to persist when the nitrogen plasma is kept on but both the gallium and indium fluxes were off. Also, if indium atoms were deposited onto the GaN surface when the nitrogen plasma source was off (producing no $\sqrt{3} \times \sqrt{3}$ reconstruction), subsequently turn- 


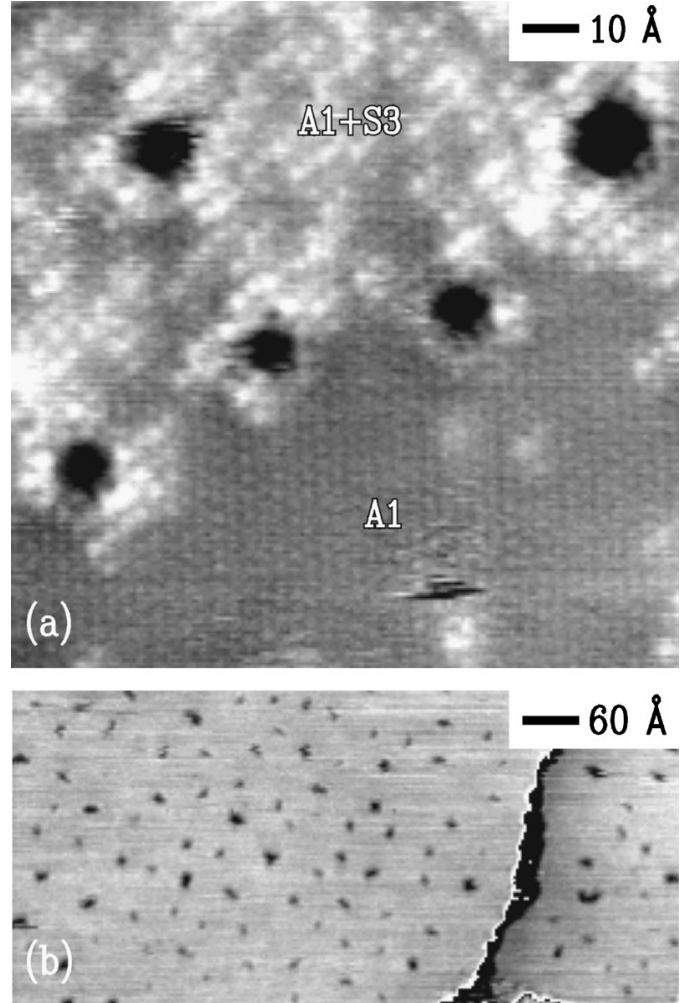

FIG. 4. STM image of $\operatorname{InGaN}(0001)$ surface: (a) surface containing 0.9 $\pm 0.2 \mathrm{ML}$ of indium. Image was acquired at a sample voltage of $+1.25 \mathrm{~V}$, and with tunnel current of $0.075 \mathrm{nA}$. Gray scale range is $0.5 \AA$. Different surface regions are labeled $\mathrm{A} 1$ and $\mathrm{A} 1+\mathrm{S} 3$. (b) Surface containing 1.4 $\pm 0.2 \mathrm{ML}$ of indium, acquired at sample voltage of $-2.0 \mathrm{~V}$ and tunnel current of $0.075 \mathrm{nA}$. The entire surface consists of the $\mathrm{A} 1+\mathrm{S} 3$ structure. A split gray scale is used, with separate terraces shown on the right- and left-hand sides of the image. A gray scale range of $2.4 \AA$ is used for both terraces.

ing on the nitrogen plasma source will make the $\sqrt{3} \times \sqrt{3}$ reconstruction appear and persist. All these experiments are carried out at the growth temperature, which was around $600{ }^{\circ} \mathrm{C}$.

In summary, we find that the $\sqrt{3} \times \sqrt{3}$ reconstruction forms under N-rich conditions, and it contains about 1/3 ML of indium atoms. It appears to consist of a structure involving indium and nitrogen atoms, on the gallium terminated GaN surface (depending on the relative indium and gallium presence on the surface, some indium atoms can go into the gallium layer). It is worth noting that growth can still be smooth with the nitrogen rich $\sqrt{3} \times \sqrt{3}$ reconstruction, implying relatively low surface diffusion barriers for this structure.

\section{STM results of InGaN(0001) surfaces under metal rich conditions}

Scanning tunneling microscopy has been used to study the structure of the InGaN(0001) surface, as shown in Fig. 4. The film shown in Fig. 4 (a) was grown at $610^{\circ} \mathrm{C}$, with $\mathrm{Ga}$ and In flux rates of $1.7 \times 10^{14}$ and $4.8 \times 10^{13} \mathrm{~cm}^{-2} \mathrm{~s}^{-1}$, respectively, well above the smooth/rough transition line. This particular film was too thin to conveniently determine its indium composition from XRD, but by comparison with other films with similar growth conditions we estimate the indium content in the bulk to be $1 \%-2 \%$. The surface was known from Auger measurements to contain 0.9 $\pm 0.2 \mathrm{ML}$ of indium. From prior theoretical work we expect the possible presence of surface structures containing In atoms either in the top surface layer or in the top two layers, ${ }^{5}$ as illustrated in Fig. 1(b). In Fig. 4(a), we see in the lower part of the image a region of uniform $1 \times 1$ corrugation. We assign the structure of this region as having indium only in the top layer, and we refer to this structure as the "A1 phase.' The bright corrugation maxima observed elsewhere in the image we attribute to In atoms in the S3 layer, since the height of the observed bright maxima, typically $0.2 \AA$ above the nominal height of the $1 \times 1$ region, is consistent with theoretical result of $0.30 \AA$ for the change in surface height induced by a second layer indium atom as discussed in the following section. The small black pits appearing on the surface appear dark (lower surface height) for both positive and negative sample bias voltage, indicating that they are some type of surface vacancy island. The pits do not grow with time, but rather, they have an equilibrium diameter of 10-20 ̊. The depth of the smallest pits seen in Fig. 2 is typically $0.7 \AA$, but this value is probably limited by the shape of the STM probe tip. For the larger pits we find a depth of $2.0 \AA$, indicating that at least one layer of atoms is missing from the surface. In surface regions containing the pits, we find $15 \%-25 \%$ indium occupation of the second layer. As discussed in the next section, we believe these pits form to relieve the strain induced by incorporation of indium in the second layer. Indeed, as can be seen from Fig. 4(a), these pits are closely related to the presence of indium in the second layer.

Figure 4(b) shows a STM image obtained from an In$\mathrm{GaN}(0001)$ film grown with similar fluxes as that in Fig. 4(a), but with slightly lower growth temperature of $600^{\circ} \mathrm{C}$. The bulk indium content of this sample is about $4 \%$, and the surface indium coverage is $1.4 \pm 0.2 \mathrm{ML}$. In this case surface regions containing pits cover practically the entire surface, with average separation between pits of about $40 \AA$. We refer to this surface phase, containing pits and partial occupation of indium in the second layer, as " $\mathrm{A} 1+\mathrm{S} 3$." The higher coverage of pits in this sample with higher indium surface concentration is consistent with the conclusion that pits are related to the indium incorporation in the second layer.

Another important feature seen in Fig. 4(a) is that the indium concentration in the second layer is higher around pits than that far from pits. This inhomogeneous surface indium concentration may contribute to the formation of the widely observed indium compositional fluctuation in the bulk InGaN. ${ }^{2,19}$ Instead of forming from bulk properties, the indium compositional fluctuation could be grown in from the inhomogeneous surface, produced by the processes of indium surface segregation ${ }^{4,5}$ and formation of a strained surface layer due to lattice mismatch between $\mathrm{InN}$ and $\mathrm{GaN}$. This strain is large because the indium surface concentration is much higher than its bulk concentration. 


\section{Theory and discussion}

The results of the previous sections are all closely related to the structure of the $\operatorname{InGaN}(0001)$ surface. In this section we will review some previous theoretical work on the structure of the InGaN(0001) surface as well as summarize recent theoretical results that support our interpretation of the STM images. We will also discuss the relationship between the surface structure and the expected diffusion mechanism for nitrogen on the In-covered surface.

Previous theoretical studies of the $\operatorname{InGaN}(0001)$ surface were performed in order to gain an understanding of indium induced morphological changes occurring on the (0001) surface. ${ }^{5,20}$ In those studies it was shown that under conditions leading to very high concentrations of indium in the films, the (0001) surface would be close to a structural instability, and that near the core of threading dislocations intersecting the surface it would be energetically favorable to form (10 $\overline{1} 1)$-faceted inverted hexagonal pyramids in order to reduce strain energy associated with the dislocation. Of course, in the present case the pits seen in the films are much smaller and are not associated with dislocations; they are an intrinsic feature of the indium induced surface reconstruction. Nevertheless, the results of the previous investigations also provide guidance for understanding the origin of the smaller pits seen in the present work. A key idea emerging from that work is that the energy of indium surface segregation is comparable to the cost of forming the surface itself. In other words, the incorporation of indium into either the bulk or the S3 layer [Fig. 1(b)], where it makes either four or three compressively strained bonds with nitrogen atoms, competes energetically with morphological changes that allow the indium to be incorporated in sites where it makes fewer, but less strained, In-N bonds. In the case of the large inverted hexagonal pit formation, the indium is incorporated onto the (1011) surfaces of the pits where it makes one or two In-N bonds. In the present case we believe that the edges of the pits in the $\mathrm{A} 1+\mathrm{S} 3$ phase exhibit sites where the indium atoms are bonded to just one or two nitrogen atoms, and the driving force stabilizing the formation of the pits is the relaxation of the surface strain associated with the indium atoms incorporated into the S3 layer. It is essential that the energy cost of forming the pits is sufficiently low so that the combination of pit formation and the resulting strain relief is energetically favorable.

Total energy calculations performed for a set of five structures having between 0 and $1 \mathrm{ML}$ of indium in the second layer establish that there is a significant amount of strain induced by substitution of indium for gallium in the second layer. These calculations were performed in a $2 \times 2$ unit cell and the second layer contained $0,1,2,3$, or 4 indium atoms. In all cases the top layer consists entirely of indium. We denote the structures in this set by specifying the total indium coverage; thus the 5/4 ML structure contains $\Theta$ $=1 / 4 \mathrm{ML}$ of indium in the second layer and $1 \mathrm{ML}$ of indium in layer 1 (the A1 layer). $\Theta$ is the indium coverage in the S3 layer. The energies of these five structures are plotted as a function of the Ga chemical potential in Fig. 5. Assuming the

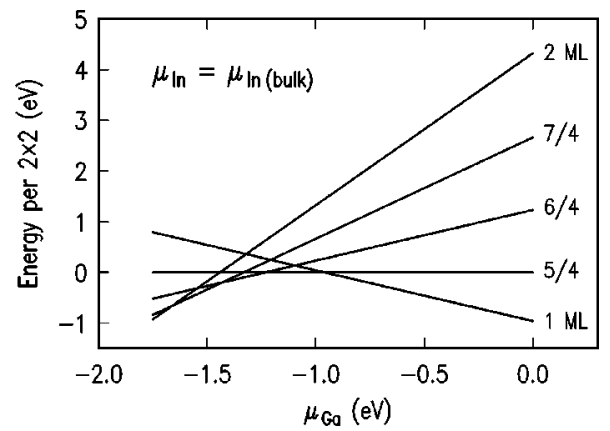

FIG. 5. Results of total energy computation for InGaN(0001) surfaces with various indium coverages, computed using a $2 \times 2$ unit cell. In all cases there is $1 \mathrm{ML}$ of indium in the top layer (the A1 layer), with the remaining indium residing in the second layer (the S3 layer).

linear elasticity theory the surface energy (per $1 \times 1$ cell) of the various $\mathrm{A} 1+\mathrm{S} 3$ surface structures can be written as

$$
E(\Theta)=E_{\mathrm{A} 1}+\left(E_{\mathrm{In}}+\mu_{\mathrm{Ga}}\right) \Theta+E_{\mathrm{In}-\mathrm{In}} \Theta^{2} .
$$

Here, $E_{\mathrm{A} 1}$ is the surface energy of the $4 / 4$ ML structure, $E_{\mathrm{In}}$ is the energy cost to incorporate a single In atom into the S3 layer (for $\mu_{\mathrm{Ga}}=0 \mathrm{eV}$ ), and $E_{\mathrm{In}-\mathrm{In}}$ is the In-In interaction energy arising from overlapping strain fields. Using the calculated surface energies we obtain $E_{\mathrm{In}}=0.86 \mathrm{eV}$ and $E_{\mathrm{In}-\mathrm{In}}$ $=0.46 \mathrm{eV}$. Higher order contributions in $\Theta$ are an order of magnitude smaller. The large positive value of $E_{\text {In }}$ implies that In can be incorporated into the S3 layer only for very Ga-deficient conditions $\left(\mu_{\mathrm{Ga}}<-0.86 \mathrm{eV}\right)$. For $\mu_{\mathrm{Ga}}$ $>-0.86 \mathrm{eV}$ it costs energy to incorporate even a single isolated indium atom into the S3 layer. Over a large range of chemical potentials $\left(-0.86 \mathrm{eV}<\mu_{\mathrm{Ga}}<0 \mathrm{eV}\right)$ the A1 phase is the energetically preferred structure. We believe this is the $1 \times 1$ structure that is seen over large areas of the surface for the low In-content film discussed in the previous section. The atomic structure of the A1 phase is indicated schematically in Fig. 6(a).

The positive value for $E_{\text {In-In }}$ is indicative of an effective repulsive interaction between the In atoms that arises because it becomes progressively more difficult to relax the compressive strains as more indium is added to the second layer. Thus, with increasing In coverage the repulsive In-In

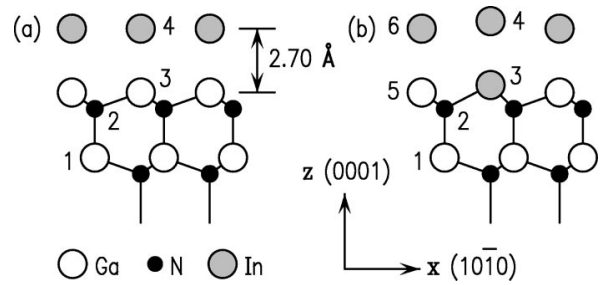

FIG. 6. (a) Schematic model of the A1 phase. The distance between the second layer gallium atoms and the first layer indium atoms is $2.70 \AA$. The distance between the layer 3 nitrogen atoms and the layer 2 gallium atoms is $0.68 \AA$. The bond angle $\theta_{123}$ is $110^{\circ}$. (b) Schematic model of the 5/4 ML structure. A vertical corrugation in the top layer is caused by the partial occupation of indium atoms in the second layer. This corrugation is $z_{4}$ $-z_{6}=0.30 \AA$. The distance between the layer 3 nitrogen atoms and the layer 2 indium atoms is $z_{3}-z_{2}=0.98 \AA$. The bond angle $\theta_{123}$ is $117^{\circ}$. 
interaction makes the In incorporation less and less favorable. In this model the gallium chemical potential has to be reduced to values below $\mu_{\mathrm{Ga}}=-\left(E_{\mathrm{In}}+2 E_{\mathrm{In}-\mathrm{In}}\right)=-1.78 \mathrm{eV}$ in order for all the $\mathrm{S} 3$ sites to be occupied by In atoms. That the In-N bonds are compressively strained, even in the 5/4 ML structure, can be seen by analyzing the atomic structure. In particular we note that the $\mathrm{Ga}-\mathrm{N}-\mathrm{In}$ bond angle $\theta_{123}$ defined in Fig. 6(b) is $117^{\circ}$ for the 5/4 ML structure. This angle increases to $119^{\circ}$ for the $8 / 4 \mathrm{ML}$ structure. The optimal value for an unstrained system would be the tetrahedral angle, $109.5^{\circ}$. Moreover, the In-N bond length is $2.13 \AA$ for the $5 / 4$ ML structure and $2.09 \AA$ for the $8 / 4 \mathrm{ML}$ structure. In calculations for bulk InN we find the In-N bond length to be 2.17 $\AA$. All these results indicate the existence of compressive strain, even for the low coverage 5/4 ML structure, and provide support for our contention that the pits form to relieve strain induced by the incorporation of indium in the second layer.

The second major theoretical result relating to this work concerns the smooth/rough transition of the surface morphology for $\operatorname{InGaN}(0001)$ compared to $\operatorname{InGaN}(000 \overline{1})$. As discussed above, the clean GaN surface, both (0001) and $(000 \overline{1})$, undergoes a smooth to rough transition as the Ga flux is reduced. It has been previously argued that this transition arises from a kinetic accumulation of nitrogen on the N-rich surfaces, such that the resulting Ga diffusivity is reduced and rough growth results. ${ }^{7}$ In the present work we have found that the effect of adding indium on the smooth/ rough transition is markedly different between the (0001) and $(000 \overline{1})$ surfaces. We believe this difference to be correlated with the different metal (In and Ga) content of the two surfaces-1 ML for the $(000 \overline{1})$ surface as pictured in Fig. 1(a), and about 2 ML for the (0001) surface as shown in Fig. 1(b). The excess metal present in the latter case may act to prevent any accumulation of nitrogen, thus preventing the associated reduction in Ga diffusivity. In addition, however, there is a separate mechanism which will act to increase the $\mathrm{N}$ diffusivity when $2 \mathrm{ML}$ of metal is present: we find that the equilibrium position of the $\mathrm{N}$ atoms in this case is between the metal layers [i.e., between A1 and S3 layers in Fig. 1(b)], and the resulting diffusivity for the $\mathrm{N}$ is significantly enhanced compared to that when the $\mathrm{N}$ resides above the top layer. ${ }^{14}$ Thus, we expect for both $\mathrm{GaN}$ and InGaN, under metal rich conditions, faster diffusion due both to the enhanced $\mathrm{N}$ diffusivity and the prevention of $\mathrm{N}$ accumulation. These mechanisms provide an interpretation of the kinetics which is in agreement with the experiment for both the clean and In-covered surfaces, of both polarity.

\section{CONCLUSION}

The effect of indium atoms on the smooth/rough transition observed during MBE growth of $\mathrm{GaN}$ and InGaN has been studied in detail. Indium atoms were found to have little effect on this smooth/rough transition when deposited onto $\mathrm{GaN}(000 \overline{1})$, but when indium is deposited on $\mathrm{GaN}(0001)$
$\mathrm{GaN}$ it is found that the gallium flux can be reduced greatly before the growth goes to rough. A $\sqrt{3} \times \sqrt{3}$ reconstruction is observed at the growth temperature. It forms and is stable under N-rich conditions. It likely consists of a structure containing indium and nitrogen atoms, residing on a gallium terminated GaN surface. InGaN(0001) surfaces under metal rich conditions were studied using STM and total energy computations. Indium is found to occupy the top two atomic layers of the crystal; incorporation in the second layer produces significant strain, leading to the formation of small pits on the surface. It is found that the indium concentration inside and around the pits is higher than that far away from the pits, which offers an alternative origin for the development of the compositional fluctuations in the InGaN bulk.

\section{ACKNOWLEDGMENTS}

This work was supported by the Office of Naval Research, Grant No. N00014-96-1-0214 (monitored by Colin Wood), and the National Science Foundation, Grant No. DMR-9615647. We thank C. D. Lee and V. Ramachandran for assistance with the MBE growth.

${ }^{1}$ S. Nakamura and G. Fasol, The Blue Laser Diode (Springer, Berlin, 1997).

${ }^{2}$ S. F. Chichibu, H. Marchand, M. S. Minsky, S. Keller, P. T. Fini, J. P. Ibbetson, S. B. Fleischer, J. S. Speck, J. E. Bowers, E. Hu, U. K. Mishra, S. P. DenBaars, T. Deguchi, T. Sota, and S. Nakamura, Appl. Phys. Lett. 74, 1460 (1999).

${ }^{3}$ F. Widmann, B. Daudin, G. Feuillet, J.-L. Rouviére, and N. Pelekanos, Appl. Phys. Lett. 73, 2642 (1998).

${ }^{4}$ H. Chen, A. R. Smith, R. M. Feenstra, D. W. Greve, and J. E. Northrup, MRS Internet J. Nitride Semicond. Res. 4S1, G9.5 (1999).

${ }^{5}$ J. E. Northrup and J. Neugebauer, Phys. Rev. B 60, 8473 (1999).

${ }^{6}$ E. J. Tarsa, B. Heying, X. H. Wu, P. Fini, S. P. DenBaars, and J. S. Speck, J. Appl. Phys. 82, 5472 (1997).

${ }^{7}$ T. Zywietz, J. Neugebauer, and M. Scheffler, Appl. Phys. Lett. 73, 487 (1998).

${ }^{8}$ V. Ramachandran, R. M. Feenstra, J. E. Northrup, and D. W. Greve, MRS Internet J. Nitride Semicond. Res. (to be published).

${ }^{9}$ V. Ramachandran, M. F. Brady, A. R. Smith, R. M. Feenstra, and D. W. Greve, J. Electron. Mater. 27, 308 (1998).

${ }^{10}$ V. Ramachandran, A. R. Smith, R. M. Feenstra, and D. W. Greve, J. Vac. Sci. Technol. A 17, 1289 (1999).

${ }^{11}$ S. Mroczkowski and D. Lichtman, Surf. Sci. 131, 159 (1983).

${ }^{12}$ R. M. Feenstra, H. Chen, V. Ramachandran, A. R. Smith, and D. W. Greve, Appl. Surf. Sci. (to be published).

${ }^{13}$ T. Böttcher, S. Einfeldt, V. Kirchner, S. Figge, H. Heinke, D. Hommel, H. Selke, and P. L. Ryder, Appl. Phys. Lett. 73, 3232 (1998).

${ }^{14}$ T. Zywietz, J. Neugebauer, J. E. Northrup, H. Chen, and R. M. Feenstra (unpublished).

${ }^{15}$ M. E. Lin, S. Strite, A. Agarwal, A. Salvador, G. L. Zhou, N. Teraguchi, A. Rockett, and H. Morkoç, Appl. Phys. Lett. 62, 702 (1993); K. Iwata, H. Asahi, S. J. Yu, K. Asami, H. Fujita, M. Fushida, and S. Gonda, Jpn. J. Appl. Phys., Part 2 35, L289 (1996); P. Hacke, G. Feuillet, H. Okumura, and S. Yoshida, Appl. Phys. Lett. 69, 2507 (1996).

${ }^{16}$ V. Ramachandran, C. D. Lee, R. M. Feenstra, A. R. Smith, J. E. Northrup, and D. W. Greve, J. Cryst. Growth 209, 355 (2000).

${ }^{17}$ A. Thamm, O. Brandt, Y. Takemura, A. Trampert, and K. H. Ploog, Appl. Phys. Lett. 75, 944 (1999).

${ }^{18}$ A. R. Smith, R. M. Feenstra, D. W. Greve, M. S. Shin, M. Skowronski, J. Neugebauer, and J. E. Northrup, Surf. Sci. 423, 70 (1999).

${ }^{19}$ Y. Narukawa, Y. Kawakami, M. Funato, Sz. Fujita, Sg. Fujita, and S. Nakamura, Appl. Phys. Lett. 70, 981 (1997).

${ }^{20}$ J. E. Northrup, L. T. Romano, and J. Neugebauer, Appl. Phys. Lett. 74, 2319 (1999). 